Tropical Journal of Pharmaceutical Research April 2015; 14 (4): 567-573

ISSN: $1596-5996$ (print); 1596-9827 (electronic)

(C) Pharmacotherapy Group, Faculty of Pharmacy, University of Benin, Benin City, 300001 Nigeria.

All rights reserved.

Available online at http://www.tjpr.org

Original Research Article

http://dx.doi.org/10.4314/tjpr.v14i4.2

\title{
Formulation and Evaluation of Liquid Loaded Tablets Containing Docetaxel-Self Nano Emulsifying Drug Delivery Systems
}

\author{
Baru Chandrasekhara Rao ${ }^{1 *}$, Suryadevara Vidyadhara ${ }^{2}$, Reddyvallam \\ Lankapalli Sasidhar ${ }^{2}$ and Yarlagadda A Chowdary ${ }^{3}$ \\ ${ }^{1}$ S.S.J College of Pharmacy, V.N Pally, Hyderabad-500 075, ${ }^{2}$ Chebrolu Hanumaiah Institute of Pharmaceutical Sciences, \\ Chowdavaram, Guntur, A.P, ${ }^{3} \mathrm{NRI}$ College of Pharmacy, Agiripalli, Nunna, Vijayawada.A.P., India \\ *For correspondence: Email: svidyadhara@gmail.com
}

Received: 24 February 2014

Revised accepted: 27 February 2015

\begin{abstract}
Purpose: To prepare and characterize tablets loaded with self-nanoemulsifying drug delivery system (SNEDDS) containing docetaxel (DTL).

Method: SNEDDS of docetaxel were prepared using various oils, surfactants, co-surfactant and solvents to improve the dissolution rate and bioavailability of the poorly water-soluble chemotherapeutic agent. The SNEDDS components were preliminarily screened for the solubility of the drug in various vehicles, miscibility of excipients, rate of emulsification and ternary phase diagrams. The tablets were prepared by direct compression process with a porous carrier, magnesium alumino-metasilicate, and subsequently loaded with SNEDDS by a simple absorption method. The tablets were then characterized for physical parameters, including tablet hardness, weight variation, disintegration, drug content and invitro drug release.

Results: Cremophor-EL, polysorbate-80 and dehydrated alcohol mixture in the ratio 85:10:5 yielded docetaxel SNEDDS with droplet size of $12.16 \mathrm{~nm}$ and polydispersity $(P D I)$ of 0.039 . Tablets with high porosity suitable for loading with SNEDDS and containg the super-disintegrants, crosscarmellose sodium and sodium starch glycolate, in a concentration of 3, 4 and $5 \%$, achieved complete dissolution of docetaxel from the tablets. In vitro release of docetaxel from SNEDDS and the tablets was similar ( $p$ $<0.05$ )

Conclusion: SNEDDS of docetaxel is a promising approach to achieving a solid dosage form of the liquid-loaded drug delivery systems for enhancing the solubility and dissolution rate of the drug, and hence also its bioavailability.
\end{abstract}

Keywords: Docetaxel, Drug carrier, SNEDDS, Self-nanoemulsifying, Solubility, Drug release, Anicancer, Surfactant, Co-surfactant

Tropical Journal of Pharmaceutical Research is indexed by Science Citation Index (SciSearch), Scopus, International Pharmaceutical Abstract, Chemical Abstracts, Embase, Index Copernicus, EBSCO, African Index Medicus, JournalSeek, Journal Citation Reports/Science Edition, Directory of Open Access Journals (DOAJ), African Journal Online, Bioline International, Open-J-Gate and Pharmacy Abstracts

\section{INTRODUCTION}

High levels of insolubility in the formulation of anticancer injections pose serious problems and challenges to formulation scientists and physicians in terms of emulsifying and suspending injectable formulations. Currently, docetaxel injection is available as SNEDDS formulation with polysorbate-80, which accommodates effective therapeutic amount in smaller amount of surfactant mix. Hence, formulation development in these areas using SNEDDS is feasible to have pharmaceutical equivalent dosage form. 
However, new technologies has been developed for sealing of soft and hard gelatin capsules [1,2]. Some formulators have focused on solid formulation of lipid systems and in particular formulation of liquids and semi-solid selfemulsifying drug delivery systems (SEDDS) [3]. Lipid-based formulation systems have become increasingly important in the formulation of lipophilic drug substances as they can facilitate the dispersion of a drug in the gastrointestinal tract resulting in enhanced oral bioavailability [4]. Self-nanoemulsifying mixture is an advanced form of nanoemulsion drug delivery systems, because water is not used and so the drug leaching from the nanosized oily droplets to the aqueous medium of the formulation during storage is minimized [6].

Generally, self-emulsifying mixtures are broadly classified according to their sizes such as: SMEDDS with droplets of emulsion size is more than $600 \mathrm{~nm}$. SMEDDS with droplets of micron sizes, i.e., lies between 100 - $150 \mathrm{~nm}$ and SNEDDS with droplets of nanosized between 10$100 \mathrm{~nm}$ [5]. Transfer of liquid lipid systems into a solid oral dosage form has been attempted by several methods such as capsule filling and spray drying [6], adsorption onto solid carriers [79] and melt granulation as well as other techniques [10]. However, a large amount of carrier is required to solidify the liquid, which subsequently results in the production of a large dosage volume [11].

The objective of this study was to develop and evaluate self-nanoemulsifying formulations containing poorly water soluble anti-mitotic chemotherapy agent, docetaxel.

\section{EXPERIMENTAL}

\section{Materials}

Docetaxel (DTL) was obtained as a gift from Aptuit Laurus Laboratories, India. Polyethoxylated castor oil (CremophornEL), polysorbate-80, PEG (polyethylene glycol) 400, propylene glycol, polyethylene glycol-15-hydroxy stearate (Solutol®HS-15), propylene glycol monocaprylate (type II) NF (Capryol囚90), glyceryl caprylate/caprate (Capmul囚MCM), Capryol-90, Capmul-MCM, lauroglycol, capryl ocaproyl polyoxyl-8 glycerides NF (Labrasol $($ ), propylene glycol dicaprylate/dicaprate NF (Labrafac®), highly purified diethylene glycol monoethyl ether EP/NF (Transcutol®P), dehydrated alcohol. Magnesium aluminum metatrisilicate and other chemicals used in the study were of analytical grade and procured locally.

\section{Solubility studies}

The solubility of docetaxel in various oils, surfactants, cosurfactants/solvents was determined. An excess amount of docetaxel was added into each vial containing $10 \mathrm{~mL}$ of selected vehicle. Then, the mixture was heated to $37{ }^{\circ} \mathrm{C}$ on water bath to facilitate the solubilization. Mixing of the systems was performed using a cyclo-mixer (CM 101, Remi, India) for $10 \mathrm{~min}$ in order to facilitate proper mixing of drug with the vehicle. The dispersions thus formed were shaken for $48 \mathrm{~h}$ in a mechanical shaker (Remi, India). After reaching equilibrium, the dispersions were centrifuged at $2500 \mathrm{rpm}$ for $20 \mathrm{~min}$ to remove undissolved docetaxel, followed by filtration through a 0.45 $\mu \mathrm{m}$ millipore membrane filter paper. The concentration of docetaxel was quantified by high performance liquid chromatography (HPLC).

\section{Construction of ternary phase diagrams}

The ternary phase diagrams of oil, surfactant, cosurfactant/solvents and water were developed using surfactant titration method. The dispersions of oil and water at certain weight ratios were titrated with surfactant/co-surfactant mix in a drop wise manner. Until visual observation of phase clarity and flowability was obtained. After the identification of self-nanoemulsion region in the phase diagrams, the SNEDDS formulations were selected at the desired component ratios $[13,14]$.

\section{Preparation of DTL-SNEDDS}

The DTL-SNEDDS were prepared by using surfactants, co-surfactant, oil/ solvent mix. The level of docetaxel was kept constant (i.e. $20 \mathrm{mg}$ ). The concentration of surfactant and other components were varied in different formulations until freely solubilized drug with stable SNEDDS were produced. The docetaxel $(20 \mathrm{mg})$ was added into the mixture and mixed using magnetic stirrer at ambient temperature until complete dissolution of docetaxel. Prepared SNEDDS were stored at room temperature and evaluated for their droplet size and polydispersity, stability and spectral analysis.

\section{Evaluation of SNEDDS}

\section{Droplet size and polydispersity (PDI)}

Docetaxel SNEDDS was diluted with distilled water (a portion diluted 1 in 25 and if clear, the 
second portion was diluted 1 in 100). The two portions were gently mixed and sonicated for 30 $s$ and allowed to settle. Clear solutions were used to measure droplet size/distribution and polydispersity was determined at $25{ }^{\circ} \mathrm{C}$ by photon correlation spectroscopy (PCS) using a Malvern Nanosizer/Zetasizer ${ }^{\circ}$ (Malvern Instruments, USA). The analysis was performed to determine mean values/ Z-Average of particle size distribution. Turbid and opaque dispersions were evaluated visually. The droplet size and PDI for the optimized formulation was recorded.

\section{Freeze-thaw cycle}

Freeze thawing was employed to evaluate the stability of drug loaded SNEEDS. Formulations were subjected to 3 freeze-thaw cycles, which included freezing at $-20{ }^{\circ} \mathrm{C}$ for $48 \mathrm{~h}$ followed by thawing to ambient room temperature $\left(37^{\circ} \mathrm{C}\right)$ for $48 \mathrm{~h}$. Nanoemulsions were observed for physical stability conditions such as phase separation or drug precipitation.

\section{Preparation of porous tablets}

The porous tablets were prepared by direct compression process. Porous adsorbent magnesium aluminum metatrisilicate (MAMS) granules was mixed with super-disintegrants and lubricated. All the ingredients were mixed in a cylindrical blender and directly compressed into tablets using 16 station rotary compression tablet press (Mini Press manufactured by ELITE Scientific Pvt Ltd). Tablets average weight of 860 mg compressed using a round $12.5 \mathrm{~mm}$ flatfaced punches were produced. The compositions of various porous tablets are given in Table 1 . The prepared tablets were subjected to evaluation of physical parameters such as thickness, hardness and disintegration test according to standard protocol.

\section{Loading procedure}

Tablets were placed in slightly excess of docetaxel-SNEDDS and allowed to absorb the liquid over a period of $24 \mathrm{~h}$. Tablets surface exposed to SNEDD was rotated after $12 \mathrm{~h}$. After the exposure, tablets surface was drained by using absorbent pad and the weight of tablets were recorded. The loaded tablets were dried at ambient conditions over a period of 3 days. Furthermore, the tablets were characterized for percentage loading by weight and for disintegration. To make maximum feasible time for loading, tablets were compressed as porous plugs with a thickness of $6.5 \pm 0.3 \mathrm{~mm}$.

\section{In vitro dissolution studies}

Dissolution rate studies of DTL-SNEDDS were performed using USP dissolution testing apparatus (type II) with rotating paddles at 100 rpm using $900 \mathrm{ml}$ of distilled water as dissolution medium. The temperature was maintained at 37 $\pm 0.5^{\circ} \mathrm{C}$ throughout the experiment. Aliquots (10 $\mathrm{ml}$ ) were withdrawn at various time intervals and same volume of dissolution medium was replaced for maintaining the constant volume of dissolution medium. Samples were filtered through $0.45 \mu$ filter and analyzed by HPLC $[15,16]$.

\section{Assay of DTL in SNEDDS}

An HPLC system (Waters HPLC 22695 Series) consisting of pump, Auto sampler, UV-Vis detector, Thermostat column compartment connected with Waters (alliance) Empower software equipped with a $230 \mathrm{~nm}$ UV-detector and YMC ODS C18, $250 \times 4.6 \mathrm{~mm}, 5 \mu$, was maintained at constant temperature of $30{ }^{\circ} \mathrm{C}$. A sample volume of $20 \mu \mathrm{L}$ DTL solution was injected. The gradient system consisted of 2 mobile phases with the compositions of mixed Phosphate buffer which consist of $1.6 \mathrm{~g}$ of potassium dihydrogen phosphate and $0.3 \mathrm{~g}$ of Dipotassium hydrogen phosphate in $1 \mathrm{~L}$ of water ( $\mathrm{pH}$ 5.80): Acetonitrile in the ratio 30: 70. The flow rate was kept constant at $1.0 \mathrm{~mL}$ per min.

Table 1: Composition of porous tablets

\begin{tabular}{|c|c|c|c|c|c|c|c|c|}
\hline \multicolumn{9}{|c|}{ Content (mg) } \\
\hline Ingredient & A1 & A2 & A3 & A4 & A5 & A6 & B1 & $B 2$ \\
\hline MAMS & 830.25 & 821.75 & 813.25 & 830.25 & 821.75 & 813.25 & 791.60 & 791.60 \\
\hline Sodium starch glycolate & 25.50 & 34.00 & 42.50 & -- & -- & -- & 42.50 & 25.50 \\
\hline $\begin{array}{l}\text { Crosscarmellose sodium } \\
\text { Magnesium stearate } \\
(0.5 \%)\end{array}$ & $4 . \overline{25}$ & $\overline{4.25}$ & $4 . \overline{25}$ & $\begin{array}{c}25.50 \\
4.25\end{array}$ & $\begin{array}{c}34.00 \\
4.25\end{array}$ & $\begin{array}{c}42.50 \\
4.25\end{array}$ & $\begin{array}{c}25.50 \\
4.25\end{array}$ & $\begin{array}{c}42.50 \\
4.25\end{array}$ \\
\hline Total weight (mg) & 860 & 860 & 860 & 860 & 860 & 860 & 860 & 860 \\
\hline
\end{tabular}




\section{RESULTS}

\section{Solubility}

Initially solubility studies were performed to identify suitable oily phase, surfactants, and cosurfactants for the development of SNEDDS of docetaxel. Because solubility of the drug is an important consideration when formulating a selfemulsifying formulations for avoiding precipitation of the drug on dilution in the in vivo. The concentration of docetaxel was quantified by HPLC. The results of solubility studies are reported in Figure 1.

\section{Ternary phase diagrams}

Based on the results of preliminary studies, ternary phase diagrams of the seven systems were constructed with the objective to study the relationship between the phase behavior and the composition also help to determine the concentration range of components for the formation of a nano emulsion. All the components were converted to weight/weight percent $(\mathrm{w} / \mathrm{w} \%)$ before constructing the phase diagrams. The bluish area enclosed in the triangle represents the region of selfemulsification (Fig 2). Within this area the SNEDDS form fine oil in water emulsion with only gentle agitation. Globule size $(<200 \mathrm{~nm})$ and PDI $(0.3<)$ were the criteria for selection of the composition.

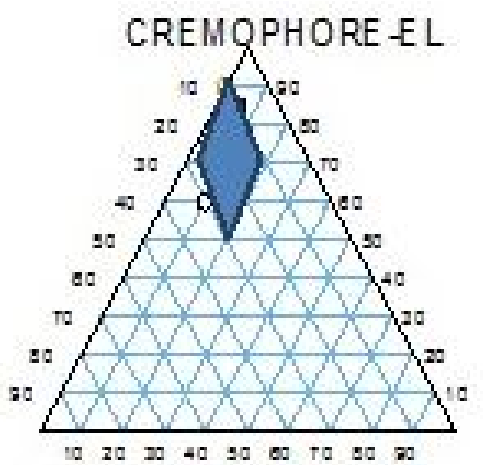

POLYSORBATE-80

ETHANOL

Fig 2: Representative ternary phase diagram indicating the efficient self-nanoemulsion region containing Cremophore-EL, Polysorbate-80 and Ethanol $(85 / 10 / 05 \%)$

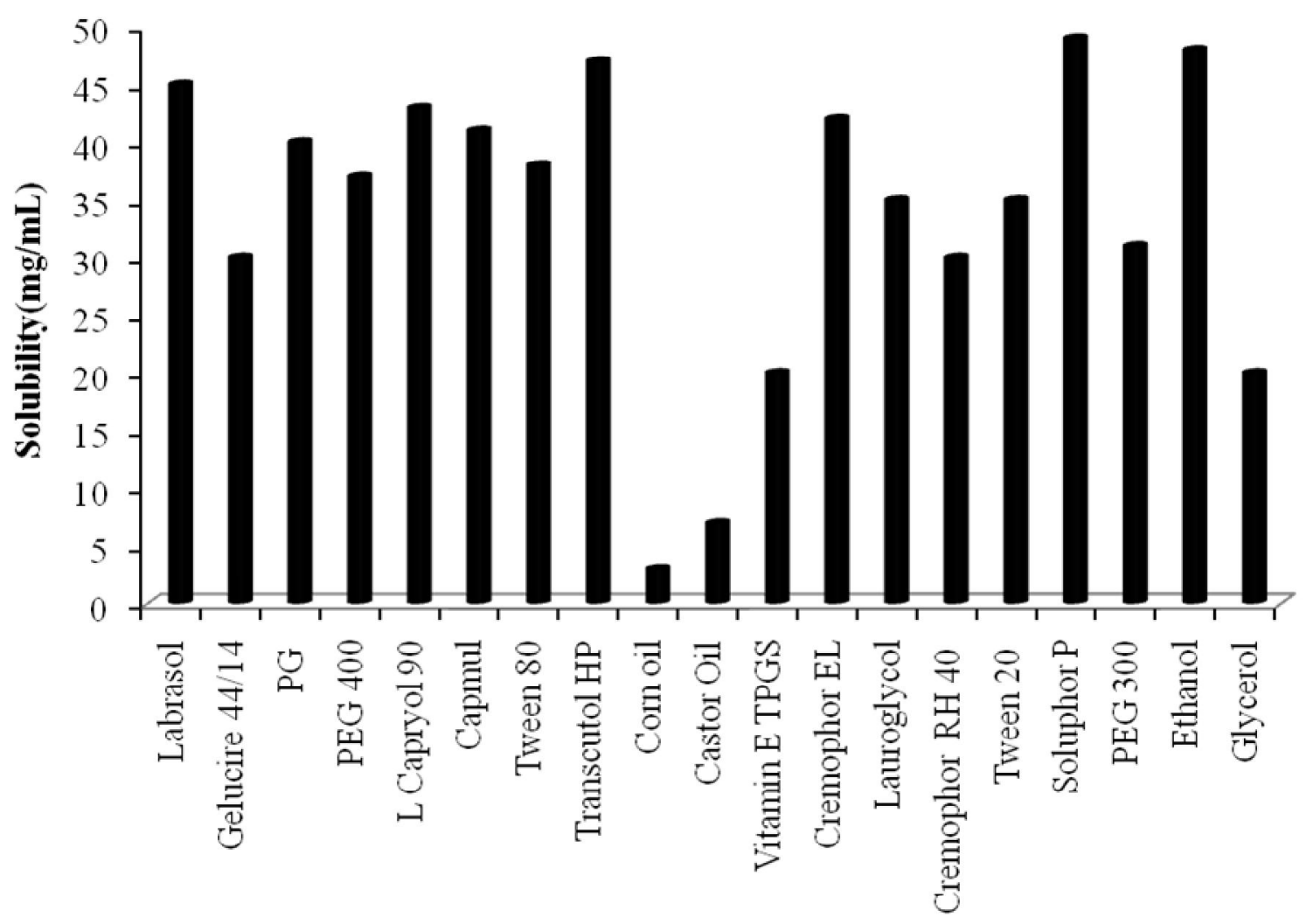

\section{Surfactant/Co surfactant/Solvent)}

Figure 1: Solubility of docetaxel in various oils, surfactants, co-surfactants and solvents 


\section{Droplet size and polydispersity}

Different surfactant systems were chosen for SNEDDS formulation. Presence of oil phase components Transcutol or Capmul in surfactant mix yielded droplet size range below $200 \mathrm{~nm}$. Due to fluidity and collision over oil and cosurfactant phases upon dilution droplet growth was observed. Replacing oil phase with solvent phase made stabilization of the nano emulsion. Here the surfactant mix which provided the rigid micelle structure and the suitable solvent proportion decreased the friction between micellar layers and resulted in stable nano emulsion. The droplet size and polydispersity values of docetaxel SNEDDS formulations are given in Table 3.

\section{Thermodynamic stability}

The selected formulations were subjected to different thermodynamic stability by using heating and cooling cycle, centrifugation and freeze thaw cycle stress tests. Those formulations, which survived thermodynamic stress tests, were taken for dispersibility test to see the visual clarity after infinite dilution. There were no physical variations observed in set of
SNEDDS developed with docetaxel, Cremophor, polysorbate- 80 and ethanol.

\section{Micrometrics}

The granules were evaluated for their micromeritic properties. The results obtained were bulk density $\left(0.615 \mathrm{~g} / \mathrm{cm}^{3}\right)$, tapped density $\left(0.510 \mathrm{~g} / \mathrm{cm}^{3}\right)$ and angle of repose $\left(18^{\circ}\right)$.

\section{Physical characteristics of SNEDDDS-loaded tablets}

Tablet weight was taken before loading and after loading as part of the characterization of loaded tablets. The tablet properties are given in Table 4.

\section{In vitro dissolution of SNEDDS-loaded docetaxel tablets}

The dissolution profiles are shown in Figure 3. Formulations $A 1, A 2, A 3$ contain sodium starch glycolate as superdisintegrant; formulations A4, A5 and A6 contain cross caremellose sodium as superdisintegrant, while formulations B1 and B2 contain the combination of both disintegrants.

Table 3: Droplet size and polydispersity (PDI) of docetaxel SNEDDS formulations

\begin{tabular}{lccc}
\hline Composition & Dilution & $\begin{array}{c}\text { Z-average } \\
\text { (d.nm) }\end{array}$ & PDI \\
\hline Polysorbate-80 +PEG-400+Transcutol-HP & $1: 25$ & 85.43 & 0.209 \\
Polysorbate-80+Soluphor-P+Capmul-MCM & $1: 100$ & 133.3 & 0.324 \\
Polysorbate-80+Glycerol+Capmul-MCM & $1: 25$ & 29.75 & 0.276 \\
Cremophor-EL+Soluphor+Capmul-MCM & $1: 100$ & 39.30 & 0.243 \\
Cremophor-EL+Glycerol+ Capmul-MCM & $1: 25$ & 96.89 & 0.434 \\
Cremophor-EL+Glycerol+Capmul-MCM & $1: 100$ & 67.8 & 0.633 \\
Cremophor-EL+Polysorbate-80+Ethanol & $1: 25$ & 10.28 & 0.873 \\
& $1: 100$ & 40.06 & 0.137 \\
& $1: 25$ & 80.93 & 0.453 \\
& $1: 100$ & 78.4 & 0.784 \\
& $1: 25$ & 98.64 & 0.305 \\
& $1: 100$ & 23.67 & 1.000 \\
\end{tabular}

Table 4: Characteristics of SNEDDS-loaded tablets

\begin{tabular}{lcccccccc}
\hline Parameter & A1 & A2 & A3 & A4 & A5 & A6 & B1 & B2 \\
\hline Mean weight & & & $860 \mathrm{mg} \pm 0.4 \mathrm{mg}$ & & & & \\
Diameter $(\mathrm{mm})$ & & & \multicolumn{7}{c}{$12.5 \pm 0.2$} & & & \\
Hardness $\left(\mathrm{kg} / \mathrm{cm}^{2}\right)$ & 2.2 & 1.8 & 2.0 & 2.4 & 2.2 & 2.2 & 2.0 & 2.2 \\
Thickness $(\mathrm{mm})$ & $6.4 \pm 0.2$ & $6.5 \pm 0.2$ & $6.5 \pm 0.2$ & $6.5 \pm 0.2$ & $6.5 \pm 0.2$ & $6.5 \pm 0.2$ & $6.5 \pm 0.2$ & $6.5 \pm 0.2$ \\
Disintegration time & $2-3$ & $1-2$ & $1-2$ & $1-2$ & $1-2$ & $\leq 1$ & $\leq 1$ & $\leq 1$ \\
(min) & & & & & & & & \\
\hline
\end{tabular}




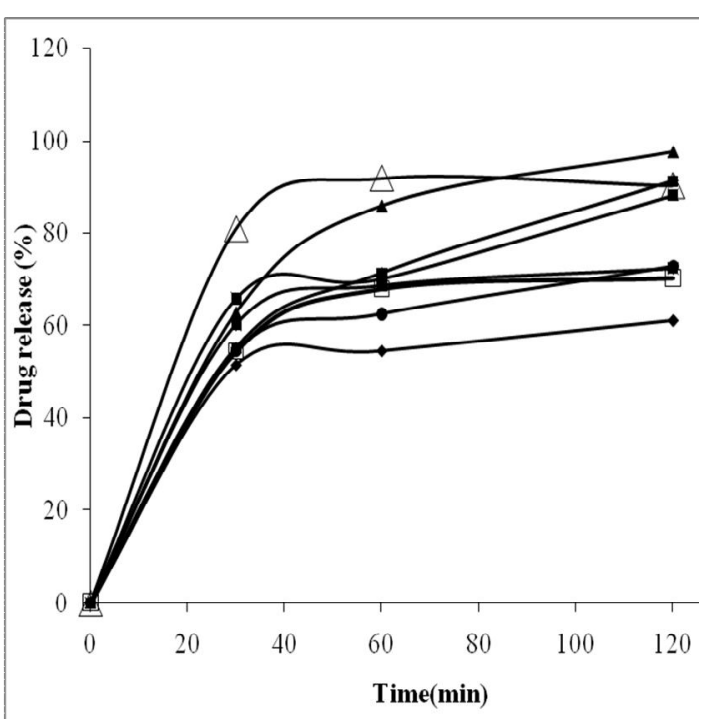

Fig 3: Dissolution profile of SNEDDS-loaded docetaxel tablets. Note: - $-A 1,-\square-A 2,-\mathbf{A}-A 3,-$ - $-\mathrm{A} 4,-$ $\bullet-A 5,-\bullet-A 6,-\square-B 1,-\Delta-B 2$

\section{DISCUSSION}

Docetaxel SNEDDS used in preparing liquidloaded tablets had a particle size of $12.16 \mathrm{~nm}$. SNEDDS dramatically enhanced the solubility of docetaxel. The results obtained in the study suggest that SNEDDS is an efficient and potential carrier for delivery of docetaxel by oral route, From the results of tablet disintegration for LLT it was found to influence the release of the loaded DTL-SMEDDS in vitro. Surfactant and cosurfactant get preferentially adsorbed at interfaces, thus reducing the interfacial energy as well as providing a mechanical barrier to coalescence, and improving the thermodynamic stability of the nanoemulsion formulation [17].

Furthermore, co-surfactants increase interfacial fluidity by penetrating into the surfactant film creating void spaces between the surfactant molecules [18]. The optimum formulation of selfnanoemulsion containing docetaxel, cremophore$\mathrm{EL}$, polysorbate- 80 and ethanol is represented by the ternary phase diagram in Figure 2. It was observed that at the dilution stage, a consistent droplet size was achieved when the polarity of docetaxel surfactant mix matches with that of water. Here, the association of oil phase with insoluble docetaxel created a high degree of non-polarity and micellar rearrangement, and thereby increased droplet size. The existence of emulsion after dilution is a promising SNEDD at micellar level as nanoemulsion with droplet size of $6-12 \mathrm{~nm}$. Porous tablets were prepared by dry granulation and along with disintegrants the micromeritics were found to be in constant range and exhibited good flow properties.
SNEDDs loaded tablets were shown dissolution rate dependant to disintegrant, in which SSG was proved better than CCS for a release and dissolution of $>95 \% \mathrm{w} / \mathrm{w}$ of docetaxel. Based on the in vitro dissolution studies Liquid loaded tablets of formulation A3 and B2 were found as optimum formulations, which were exhibited high dissolution rate than compared to the others. For these two formulations dissolution rate was accelerated by content of disintegrant. From loaded magnesium aluminometasilicate MAMS powder (A3), almost $97.7 \%$ was released. The idea has previously been investigated for tablets prepared from magnesium aluminometasilicate (MAMS) as carriers of chemical reagents and catalysts [12].

\section{CONCLUSION}

Docetaxel tablet formulations incorporating SNEDDS for enhancement of the solubility and dissolution rate of a poorly soluble drug is feasible using the approach presented in this study. Furthermore, appropriate inclusion of surfactant and co-surfactant improves the thermodynamic stability of the nanoemulsion formulation.

\section{REFERENCES}

1. Cole ET, Cade D, Benameur $H$. Challenges and opportunities in the encapsulation of liquid and semisolid formulations into capsules for oral administration. Adv Drug Deliv Rev, 2008; 60: 747756.

2. Bergstrom DH, Waranis RP, Rahman MS. Capsules, soft. In: Swarbrick J, Boylan JC, Eds. Encyclopedia of Pharmaceutical Technology. 2nd edn. New York: Marcel Dekker, 2002; pp 317-327.

3. Marchaud D, Hughes S. Solid dosage forms from selfemulsifying lipidic formulations. Pharm Eur, 2008; 1: 46-49.

4. Porter CJ, Trevaskis NL, Charman WN. Lipids and lipidbased formulations: optimizing the oral delivery of lipophilic drugs. Nat Rev Drug Discov, 2007; 6(3): 231-248.

5. Pouton CW. Formulation of poorly water-soluble drugs for oral administration: physicochemical and physiological issues and the lipid formulation classification system. Eur J Pharm Sci, 2006; 29: 278-287.

6. Yi $T$, Wan J, Xu $H$, Yang $X$. A new solid selfmicroemulsifying formulation prepared by spraydrying to improve the oral bioavailability of poorly water soluble drugs. Eur J Pharm Biopharm, 2008; 70: 439-444. 
7. Patil P, Joshi P, Paradkar A. Effect of formulation variables on preparation and evaluation of gelled selfemulsifying drug delivery system (SEDDS) of Ketoprofen. AAPS PharmSciTech, 2004; 5: 43-50.

8. Dixit RP, Nagarsenker MS. Self-nanoemulsifying granules of ezetimibe: design, optimization and evaluation. Eur J Pharm Sci, 2008; 35: 183-192.

9. Nazzal S, Nutan M, Palamakula A, Shah R, Zaghloul AA, Khan MA. Optimization of a self-nanoemulsified tablet dosage form of Ubiquinone using response surface methodology: effect of formulation ingredients. Int $J$ Pharm, 2002; 240: 103-114.

10. Jannin V, Musakhanian J, Marchaud D. Approaches for the development of solid and semi-solid lipid-based formulations. Adv Drug Deliv Rev, 2008; 60: 734 746.

11. Bansal T, Mustafa G, Khan ZI, Ahmad FJ, Khar RK, Talegaonkar S. Solid self-nanoemulsifying delivery systems as a platform technology for formulation of poorly soluble drugs. Crit Rev Ther Drug Carrier Syst, 2008; 25: 63-116.

12. Ruhland $T$, Nielsen SD, Holm $P$, Christensen $\mathrm{CH}$. Nanoporous magnesium aluminometasilicate tablets for precise, controlled, and continuous dosing of chemical reagents and catalysts: applications in parallel solution-phase synthesis. J Comb Hem, 2007; 9: 301-305.

13. Shah N, Carvajal M, Patel C, Infeld M, Malick A. Selfemulsifying drug delivery systems (SEDDS) with polyglycolyzed glycerides for improving in vitro dissolution and oral absorption of lipophilic drugs. Int J Pharm, 1994; 106: 15-23.

14. Matuszewska B, Hettrick L, Bondi J, Storey $D$. Comparative bioavailability of L-683,453, a 5areductase inhibitor, from a self-emulsifying drug delivery system in beagle dogs. Int J Pharm, 1996; 136: 147-154.

15. Singh B, Singh $S$. A comprehensive computer program for study of drug release kinetics from compressed matrices. Indian J Pharm Sci 1998; 60: 358-362.

16. Singh $B$, Kaur $T$, Singh $S$. Correction of raw dissolution data for loss of drug during sampling. Indian J Pharm Sci 1997; 59: 196-199.

17. Reiss $H$., Entropy-induced dispersion of bulk liquids. $J$ Colloid Interface Sci, 1975; 53: 61-70.

18. Constantinides PP, Scalart JP, Formulation and physical characterization of water-in-oil microemulsions containing long-versus medium-chain glycerides. Int $\mathrm{J}$ Pharm. 1997; 158: 57-68. 